\title{
Accurate Banded Graph Cut Segmentation of Thin Structures Using Laplacian Pyramids
}

\author{
Ali Kemal Sinop and Leo Grady \\ Department of Imaging and Visualization \\ Siemens Corporate Research \\ Princeton, NJ, USA \\ ali.sinop. extasiemens.com, \\ leo.grady@siemens.com
}

\begin{abstract}
The Graph Cuts method of interactive segmentation has become very popular in recent years. This method performs at interactive speeds for smaller images/volumes, but an unacceptable amount of storage and computation time is required for the large images/volumes common in medical applications. The Banded Graph Cut (BGC) algorithm was proposed to drastically increase the computational speed of Graph Cuts, but is limited to the segmentation of large, roundish objects. In this paper, we propose a modification of BGC that uses the information from a Laplacian pyramid to include thin structures into the band. Therefore, we retain the computational efficiency of BGC while providing quality segmentations on thin structures. We make quantitative and qualitative comparisons with BGC on images containing thin objects. Additionally, we show that the new parameter introduced in our modification provides a smooth transition from BGC to traditional Graph Guts.
\end{abstract}

\section{Introduction}

The Graph Cuts method of interactive segmentation [1] has become very popular in recent years due to its behavioral and theoretical properties. This method performs at interactive speeds for smaller images/volumes, but an unacceptable amount of computation time is required for the large images/volumes common in medical applications. Consequently, the Banded Graph Cuts algorithm (BGC) [2] was proposed in an attempt to preserve the segmentation quality of Graph Guts while drastically increasing the computational speed. Although BGC performs well when the segmentation target is a large, blob-like object, the segmentation quality breaks down when the image contains thin structures. Since thin structures are ubiquitous in medical images (typically in the form of vessels), the BGC algorithm must be modified in order to apply to this problem domain. We present a modification of the BGC algorithm that preserves its computational advantages, but produces segmentations much closer to Graph Cuts when the segmentation target contains thin structures. This modification is based upon the use of a difference image when coarsening to identify thin structures that may be included into the band during the segmentation.

Graph Cuts has risen to become an extremely popular algorithm for image segmentation (although we will employ the generic term "image" in this paper, we intend this 
term to be understood as pertaining to data volumes as well). The Graph Cuts algorithm inputs two user-defined "seeds" (or seed groups) indicating samples of the foreground object and the background. The algorithm then proceeds to find the max-flow/min-cut between these seeds, viewing the image as a graph where the pixels are associated with nodes and the edges are weighted to reflect image gradients. Although standard maxflow/min-cut routines [3] may be used for this computation, faster specialty algorithms for the domain of image segmentation have been developed [4]. Part of the popularity of the Graph Cuts algorithm also stems from the statistical interpretation of the approach as the minimization of a certain Markov Random Field [56]. Additionally, the segmentation results are straightforward to predict and intuitive to use since the algorithm always finds the segmentation boundary at the location of the minimum cut (or surface, under the interpretation of [7]).

The max-flow/min-cut algorithm introduced by [4] makes a great computational advance over the conventional methods for computing max-flow/min-cut. However, for high-resolution images or medical volumes, the computational burden is still too large for the algorithm to operate at an interactive speed. For example, it was reported in [2] that over six minutes were required to segment a medical volume of size $256 \times 256 \times 185$. This large computational burden for the Graph Cuts algorithm prompted the introduction of the BGC algorithm [2]. The goal of the BGC algorithm was to provide the same cut (segmentation) of the native Graph Cuts algorithm by introducing a multilevel scheme for the computation. Although the BGC algorithm is not guaranteed to provide the minimum graph cut, it was convincingly shown in [2] that the (near) minimal graph cut was returned for practical problems in which the segmentation target was "blob-like". However, the authors of [2] state (and demonstrate) clearly that the BGC algorithm is inappropriate for thin objects. Unfortunately, the medical imaging domain frequently contains thin segmentation targets in the form of vascular structures. Despite this limitation to "blob-like" segmentation targets, the BGC algorithm produces computational speeds over an order of magnitude faster than those obtained when employing conventional Graph Cuts (i.e., using the algorithm of [4] on the full graph).

The BGC algorithm is not the only approach to increasing the computational efficiency of Graph Cuts. The Lazy Snapping algorithm of [8] performs a presegmentation grouping of the pixels using a watershed transform [9] and treats each watershed basin as a supernode. Graph Cuts is then run on the graph of supernodes. By drastically reducing the number of nodes in the graph, this approach greatly increases the speed of Graph Cuts in practice. However, the computational savings are more difficult to predict with this approach since the number of supernodes is highly image dependent. Additionally, the authors of this approach offer no characterization of when to expect this coarsening approach to succeed or fail to produce the same cut given by full-resolution Graph Cuts.

We propose a modification of the BGC algorithm that allows for the accurate segmentation (relative to full-resolution Graph Cuts) of thin objects while preserving the computational advantages of BGC. The modification is born of the observation that thin structures are lost as a result of the coarsening operation involved in BGC. Therefore, we employ the idea of a Laplacian pyramid [10] to recover this lost information and extend the band such that high-threshold pixels are included. In this manner, the 
additional computational burden is slight but thin structures are recovered. The extra computational burden comes only from considering a small number of additional pixels in the band and by computation of the Laplacian pyramid, which need not be computed explicitly and therefore requires simply an additional linear-time pass over the image.

This paper is organized as follows: Section 2 gives details of the method and implementation, Section 3 gives results compared to BGC and full-resolution Graph Cuts. Finally, Section 4 offers concluding statements.

\section{Method}

We begin by first introducing the graph structures used in this algorithm. Let $(P, I)$ be an N-D image on a finite discrete set $P$ with values in $R^{N}$ and a mapping from this set to a value $I(p)$ in some value space. In this paper, we assume that $I^{0}=I$ which is the base image. For an image $I$, we can construct the associated undirected weighted graph $G=(V, E, W)$ consisting of nodes (vertices) $v \in V$, edges $e \in E$ and nonnegative weights $w \in W$ with a specified neighborhood structure. In our setting, each node is identified with one pixel and a 4-connected (6-connected in dimension 3) lattice structure of edges is assumed. For a set of images $\left\{I^{0}, \cdots, I^{k}\right\}$, a separate graph is constructed for each level, producing a set of graphs $\left\{G^{0}=\left(V^{0}, E^{0}, W^{0}\right), \cdots, G^{k}=\right.$ $\left.\left(V^{k}, E^{k}, W^{k}\right)\right\}$.

We begin by reviewing the BGC algorithm of [2] in more detail before proceeding to the proposed modifications via a Laplacian pyramid. For an input image, $I^{0}$, the BGC algorithm produces an image pyramid, $\left\{I^{1}, \cdots, I^{K}\right\}$ for a predetermined number of levels, $K$, via downsampling by a factor of two in each dimension, indicated here by the downsampling operation $D(\cdot)$, e.g., $I^{1}=D\left(I^{0}\right)$. The Graph Cuts algorithm of [4] is then applied to the coarsest-level image, $I^{K}$, to produce a minimum cut on the coarse level that assigns pixels to sets representing foreground, $F^{K} \subset V^{K}$, and background, $B^{K} \subset V^{K}$, such that $F^{K} \cup B^{K}=V^{K}$ and $F^{K} \cap B^{K}=\emptyset$. Let $\partial F^{K}$ be the boundary of the foreground segmentation defined as $\partial F^{K}=\left\{v_{i} \in\right.$ $F^{K-1} \mid \exists v_{j} \in B^{K-1}$ s.t. $\left.\left\|v_{i}-v_{j}\right\| \leq 1\right\}$, where $\|\cdot\|$ is taken to represent a Euclidean distance of the node (pixel) coordinates. This coarse-level segmentation boundary is then upsampled to level $(K-1)$, indicated by the upsampling operation $U(\cdot)$, e.g., $\partial F^{K-1}=U\left(\partial F^{K}\right)$. This upsampled segmentation boundary is modified by dilating the segmentation boundary by a distance $d$ to include a "band" of unsegmented pixels. The BGC approach then proceeds to treat the inside of the band as foreground seeds and the outside of the band as background seeds and compute the graph cut inside the band. Formally, this may be described by forming three sets of nodes on the coarse level, $F_{*}^{K-1} \subset F^{K-1}, B_{*}^{K-1} \subset B^{K-1}, Q^{K-1} \subset V^{K-1}$, where $F_{*}^{K-1}$ is the set of foreground nodes on the interior of the band, $B_{*}^{K-1}$ is the set of background nodes outside the band and $Q^{K-1}$ is the set of nodes inside the band (the "questionable" nodes). For a given $d, Q^{K-1}=\left\{v_{i} \in V^{K-1} \mid \exists v_{j} \in U\left(\partial F^{K}\right)\right.$ s.t. $\left.\left\|v_{i}-v_{j}\right\| \leq d\right\}$. Note that the (coarsened) locations of the seed pixels are never allowed to belong to the questionable set $Q^{k}$. The BGC then performs a second max-flow/min-cut computation on $I^{K-1}$ where the nodes in $F_{*}^{K-1}$ are treated as source seeds and the nodes in $B_{*}^{K-1}$ are treated as background seeds. This operation is then continued to the next level until the fine-resolution level is reached and a fine-level segmentation is obtained. 


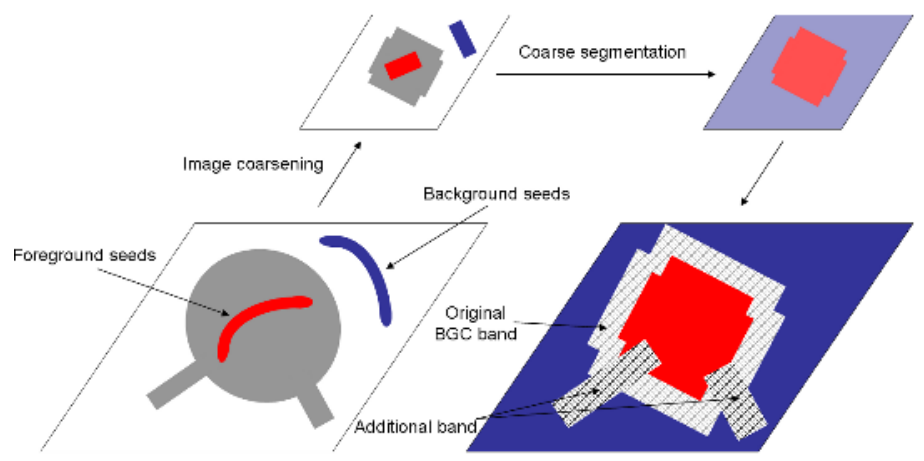

Fig. 1. Overview of the algorithm. An initial circle with two thin attached structures (e.g., vessels) is to be segmented using the foreground seeds (red) and background seeds (blue). First, the image/seeds are coarsened, during which the thin structures are lost. The segmentation is then performed on the coarse level. The resulting segmentation is then upsampled and the original BGC band is computed (indicated by the gray crosshatched area). In our approach, this band is augmented to include the thin structures (indicated by the black crosshatched area) as found by pixels with a large magnitude in the difference image of the Laplacian pyramid.

The Laplacian pyramid introduced by Burt and Adelson [10] preserves the fine-level detail by storing a second image, $S^{k}$, defined as $S^{k}=I^{k}-U\left(D\left(I^{k}\right)\right)$. This subtraction image preserves the fine details at level $k$ obscured by the downsampling and upsampling operations. Since this image preserves the fine details, we may employ these values to introduce additional nodes into the band in BGC. Specifically, we introduce a threshold, $\alpha$, and modify the definition of $Q^{k}$ given above for the "band nodes" to state $Q_{*}^{K-1}=\left\{v_{i} \in V^{K-1} \mid S^{K-1}\left(v_{i}\right) \geq \alpha\right\} \cup Q^{K-1}$ and then remove all the nodes in $Q_{*}^{K-1}$ which are not in the same connected component with the original band $Q^{K-1}$. Here $S^{k}\left(v_{i}\right)$ is taken to indicate the magnitude of the subtraction image at node (pixel) $v_{i}$. The parameter $\alpha$ may be interpreted as an "interpolation" parameter between the BGC algorithm $(\alpha=\infty)$ and the standard Graph Cuts algorithm $(\alpha=0)$. In Section 3 we show that an $\alpha$ setting much closer to BGC preserves the speed advantages of this approach but setting $\alpha<\infty$ allows the recovery of thin structures previously missed in the BGC algorithm. We note that the image $S$ need not be computed or stored explicitly, since for each pixel, we can compute the associated Laplacian value online in constant time.

Also assignment of nodes as foreground or background is changed, too. Since the band may disconnect $F^{K}$ and $B^{K}$, the final $F_{*}^{K-1}$ and $B_{*}^{K-1}$ are taken as the connected components containing foreground and background seeds respectively.

Figure 1 illustrates the entire process. An initial circle with two thin attached structures (e.g., representing an idealized organ fed with blood vessels) is to be segmented using the indicated seeds. This image is coarsened and segmented, but the "vessels" are not properly segmented due to the coarsening process. When upsampling, the band (indicated by a gray crosshatch) is augmented by the pixels crossing threshold $\alpha$ (indicated by the black crosshatch). Now, when the segmentation is performed the "vessels" will be included. Figure 2 gives an example of the process for a real image, with its corresponding subtraction image. 


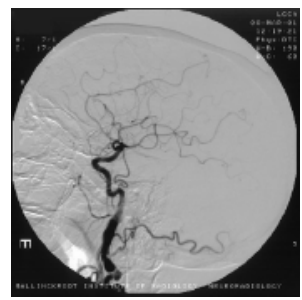

(a) Original image

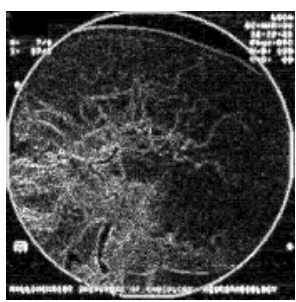

(b) Subtraction image

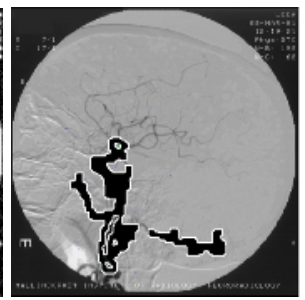

(c) Unaugmented band

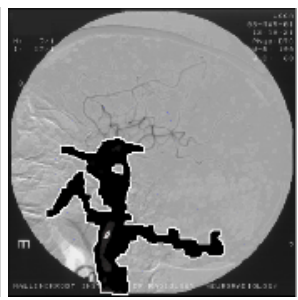

(d) Augmented band

Fig. 2. Construction of a segmentation band. (a) The original image to be segmented (seeds not shown). (b) The subtraction (Laplacian pyramid) image obtained from upsampling the downsampled image and subtracting it from the original. Note that thin (high frequency) structures are strongly represented. (c) The unmodified band resulting from an upsampled segmentation. (d) The segmentation given after augmenting the band with the high-magnitude values in the subtraction image (b).

In accordance with [2], our graph on all levels was taken as a 4-connected lattice with weights given by $w_{i j}=\exp \left(-\frac{\left(I_{i}-I_{j}\right)^{2}}{2 \sigma^{2}}\right)$. We define the upsampling and downsampling functions as $\operatorname{Up}(I)=\uparrow(I \otimes w)$ and $\operatorname{Down}(I)=(\downarrow I) \otimes w$. Here $w$ is a Gaussian filter with $\sigma=1.0, \otimes$ denotes convolution, $\uparrow$ samples odd pixels and $\downarrow$ samples each pixel twice.

\section{Results}

The validation of our augmented BGC algorithm addresses the following questions:

1. Does our augmentation of BGC perform qualitatively better on images containing thin structures (with respect to full-resolution GC)?

2. Does our augmentation of BGC perform quantitatively better on images containing thin structures (with respect to full-resolution GC)?

3. What is the tradeoff of speed/accuracy obtained by setting different values of $\alpha$ ?

The experiments were performed for both 2D and 3D data.

Figure 3 and Figure 4 address the issue of qualitative comparison for 2D and 3D data respectively. In Figure 3 two images containing vascular structures were given foreground/background seeds such that a full-resolution GC produces good segmentations. The images were then segmented using BGC and our augmented BGC using three levels. As indicated by the authors of BGC, the algorithm is unable to accurately find small/thin structures, especially when the structures are distant from the seed locations. In contrast, our augmented BGC recovers the full-resolution GC segmentations at speeds only marginally decreased from than the high-performance BGC. Both images had resolution $512 \times 512$. The same experiment was performed in 3D on an aorta and a left atrium segmentation target, with volumes $256 \times 224 \times 32$ and $124 \times 184 \times 185$, respectively. In both cases, the augmented BGC was able to recover the full resolution GC solution exactly, even though additional levels were used (i.e., another coarsening step was applied before running GC). 


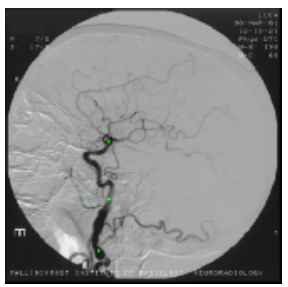

(a) Original image

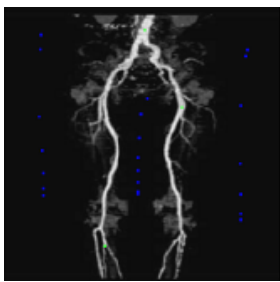

(e) Original image

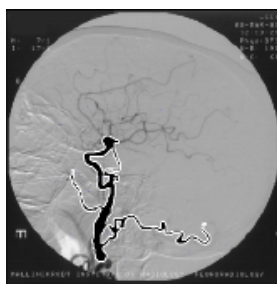

(b) Graph Cut

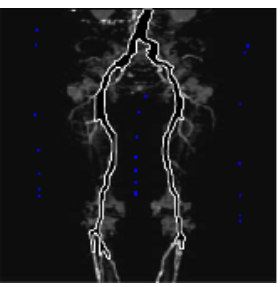

(f) Graph Cut

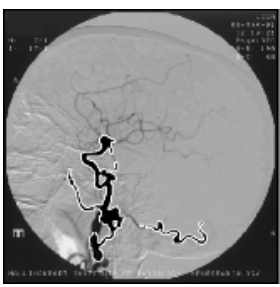

(c) BGC

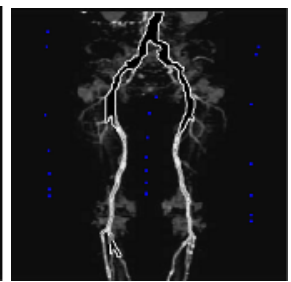

(g) BGC

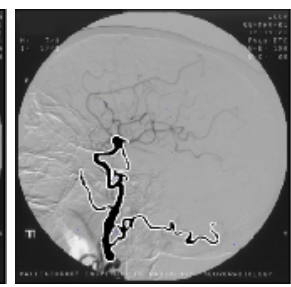

(d) Augmented BGC

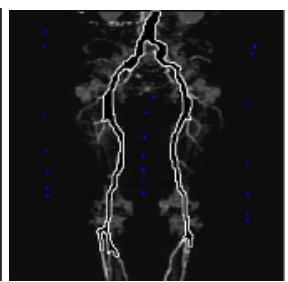

(h) Augmented BGC

Fig. 3. Qualitative comparison of BGC and augmented BGC (with respect to full-resolution GC) on two 2D images contained elongated structures. (a,e) Original images. (b,f) Full-resolution Graph Cuts segmentation. (c,g) Banded Graph Cuts solution with three levels - Note that many elongated structures are incorrectly segmented. (d,h) Augmented Banded Graph Cut solution with three levels.

A quantitative analysis of the augmented BGC was combined with an investigation into the role of the new $\alpha$ parameter. We began with a set of six number of $2 \mathrm{D}$ images containing vessels or other elongated structures. In each image, sufficient seeds were given to the full resolution GC algorithm in order to produce a quality segmentation of the elongated target. For each image, the segmentation was then performed using BGC with 2-3 levels and augmented BGC using 3 levels. The $\alpha$ parameter was then varied between $\{0-\max S\}$ and the speed/memory was tracked with respect to the segmentation accuracy. The segmentation accuracy was computed with respect to full resolution Graph Cuts by histogram intersection. Figure 3 shows the mean values with an error bar indicating one standard deviation in both speed/memory and accuracy. In these plots, the three dots corresponding to BGC indicate the use of zero coarse levels (i.e., fullresolution GC) and one or two coarse levels. Two qualities of the augmented Graph Cuts are portrayed in Figure 3. 1) The $\alpha$ parameter provides a smooth transition from the BGC algorithm to full-resolution GC, 2) By setting $\alpha$ close to zero, large gains can be made over BGC in accuracy, for mild costs in speed/memory. This experiment was repeated a second time for an aorta volume illustrated in Figure 4

Although the increases in accuracy appear less drastic than in the $2 \mathrm{D}$ case, this is simply because the number of voxels involved in the elongated pieces is very small compared to the total number of voxels in the object. However, as shown in Figure 4 the qualitative difference in accuracy is significant. Note that the augmented BGC with $\alpha=0$ requires more time than the full resolution Graph Cuts, since the coarsening, etc. become wasted, time-consuming, operations in this case. Similarly, another experiment was performed on a set of three left atrium datasets. Although the sizes of the data 


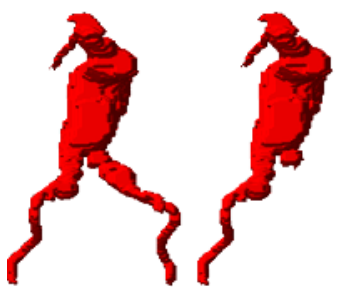

(a) Full GC

(b) BGC result

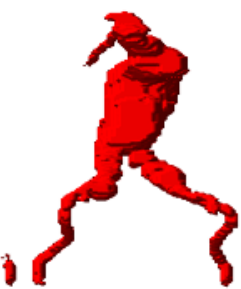

(c) Augmented BGC

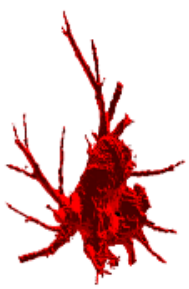

(d) Full GC

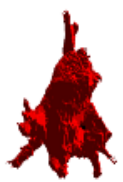

(e) BGC Result

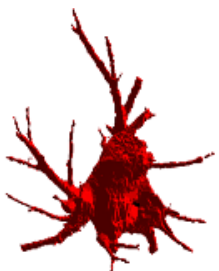

(f) Augmented BGC

Fig. 4. Qualitative comparison of the full resolution Graph Cuts, the BGC algorithm and the augmented BGC algorithm on volumetric data containing an aorta (top row) and a left atrium (bottom row). (a,d) The full resolution Graph Cuts result, requiring 10s and 300Mb of memory (a) and $40 \mathrm{~s} / 700 \mathrm{Mb}(\mathrm{d})$. (b,e) Banded Graph Cuts results with the same seeding using one coarse level, requiring 3s/36Mb (b) and $8 \mathrm{~s} / 86 \mathrm{Mb}(\mathrm{e})$. (c,f) Augmented Banded Graph Cuts results using two coarse levels, requiring $3 \mathrm{~s} / 33 \mathrm{Mb}(\mathrm{c})$ and $15 \mathrm{~s} / 216 \mathrm{Mb}(\mathrm{f})$.

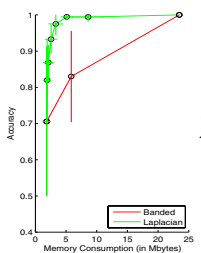

(a) 2D angiography images

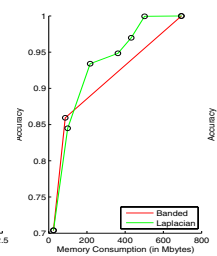

(b) Aorta dataset
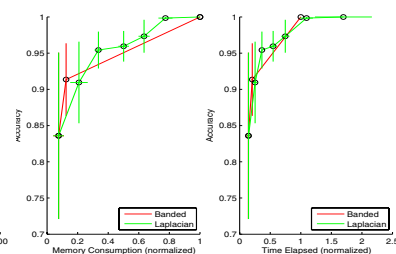

(c) Left Atrium datasets

Fig. 5. Memory/time and accuracy tradeoff plots. The horizontal and vertical error bars represent one standard deviation in the corresponding axis. In all plots, the red line indicates the Banded Graph Cuts approach with three points corresponding to two coarse levels, one coarse level and zero coarse levels (i.e., full resolution Graph Cuts). The green line indicates the performance of the augmented Banded Graph Cuts approach with three levels, using an $\alpha$ parameter from $\{0-\max S\}$.

volumes were the same, the volume of the left atrium in each case was different and therefore the memory consumption and time elapsed were normalized to unity. In this case, the bars show one standard deviation in the corresponding axis. The quantitative improvement in accuracy may seem slight, due to the fact that the inside of heart has also high Laplacian values. To remedy this problem, the Laplacian band may be forced to stay outside of the foreground region.

\section{Conclusion}

The Graph Cuts algorithm has become a very popular algorithm in recent years for interactive segmentation. However, the speed/memory restrictions of this approach prevents its interactive use on many modern medical datasets, due to their size. For this purpose, the Banded Graph Cuts algorithm was introduced to alleviate the speed/memory concerns while largely providing the same segmentation as standard Graph Cuts. However, the BGC algorithm is unable to accurately handle segmentation tasks involving 
elongated structures, which are very common in medical segmentation tasks. In this paper we introduced the Augmented Band Graph Cuts approach that preserves the positive speed/memory qualities of BGC, while allowing for an accurate result when the segmentation task involves elongated structures. Specifically, the difference information from a (simulated) Laplacian pyramid is used to add to the segmentation band at subsequent levels in order to place elongated structures within the space of considered segmentations for the fine level Graph Cuts. Our proposal to augment Banded Graph Cuts with information from a (simulated) Laplacian pyramid was studied from the standpoint of qualitative and quantitative improvement and the role of the introduced $\alpha$ parameter. These studies were carried out on both 2D and 3D datasets containing segmentation targets with an elongated object. The studies concluded that it was possible to obtain nearly the same increase in speed/memory as was determined by the BGC algorithm, but to simultaneously achieve a segmentation much closer to the full-resolution Graph Cuts. Therefore, we can conclude that the Banded Graph Cuts algorithm should always use an augmented band, since the computational increase is negligible, while the accuracy increase is significant when elongated structures are present in the image.

\section{References}

1. Boykov, Y., Jolly, M.P.: Interactive graph cuts for optimal boundary \& region segmentation of objects in N-D images. In: Proc. of ICCV 2001. (2001) 105-112

2. Lombaert, H., Sun, Y., Grady, L., Xu, C.: A multilevel banded graph cuts method for fast image segmentation. In: Proceedings of ICCV 2005. Volume I., Bejing, China, IEEE (2005) 259-265

3. Gibbons, A.: Algorithmic Graph Theory. Cambridge University Press (1989)

4. Boykov, Y., Kolmogorov, V.: An experimental comparison of min-cut/max-flow algorithms for energy minimization in vision. IEEE PAMI 26(9) (2004) 1124-1137

5. D. Greig, B.P., Seheult, A.: Exact maximum a posteriori estimation for binary images. Journal of the Royal Statistical Society, Series B 51(2) (1989) 271-279

6. Boykov, Y., Veksler, O., Zabih, R.: A new algorithm for energy minimization with discontinuities. In Pelillo-M., H.E.R., ed.: Energy Minimization Methods in Computer Vision and Pattern Recognition. Second International Workshop, EMMCVPR'99, York, UK, 26-29 July 1999. (1999) 205-220

7. Boykov, Y., Kolmogorov, V.: Computing geodesics and minimal surfaces via graph cuts. In: Proceedings of Iternational Conference on Computer Vision. Volume 1. (2003)

8. Li, Y., Sun, J., Tang, C., Shum, H.: Lazy snapping. In: Proceedings of ACM SIGGRAPH 2004. (2004) 303-308

9. Roerdink, J., Meijster, A.: The watershed transform: Definitions, algorithms, and parallellization strategies. Fund. Informaticae 41 (2000) 187-228

10. Burt, P.J., Adelson, E.H.: The Laplacian pyramid as a compact image code. IEEE Transactions on Communications COM-31(4) (1983) 532-540 